\title{
Locked-in: don't judge a book by its cover
}

\section{Bruno, ${ }^{1}$ J L Bernheim, ${ }^{2}$ C Schnakers, ${ }^{1}$ S Laureys ${ }^{1}$}

Amyotrophic lateral sclerosis (ALS; also called motor neuron disease) is a devastating medical condition that progressively robs patients of their ability to move, speak and eventually breathe. At present, many physicians are hesitant to propose tracheostomy and respiratory support in the terminal phase of ALS. In accordance with the principle of patient autonomy, physicians should respect the right of the ALS patient to accept or refuse any treatment, including mechanical ventilation. Also, in environments where euthanasia or physician-assisted death is legal, such requests can be acceptable. At least two conditions are necessary for full autonomy. To have a claim on full autonomy, people need to have intact cognitive abilities, and to exercise this right they must be able to communicate. In the past, the first condition was in doubt (indeed, overlap with other neurodegenerative diseases is sometimes suspected and some patients with ALS are thought to have associated frontotemporal dementia) and the second was severely compromised in patients with devastating motor impairment (communication being limited to the twitch of a finger or the blink of an eye).

In this issue of $J$ Neurol Neurosurg Psychiatry, Lakerveld and colleagues ${ }^{1}$ investigated cognition in 11 patients with late stage ALS (see page 25). They showed preserved cognitive functioning (ie, language, executive function, intelligence, learning and long term memory) compared with healthy controls. Assessments were exclusively based on a "yes-no" response mode. Because of the absence of verbal and motor communication, the neuropsychological assessment of these patients is complicated, and adapted testing is needed. By using a "yes-no" response mode based on the remaining motor abilities of the patient, this study proves the possibility of assessing patients with minimal motor capacities.

${ }^{1}$ Coma Science Group, Cyclotron Research Centre and Neurology Department, University of Liège, Liège,

Belgium; ${ }^{2}$ Human Ecology Department, Free University Brussels, Brussels, Belgium

Correspondence to: Professor S Laureys, Coma Science Group, Cyclotron Research Centre and Neurology Department, University of Liège, Sart Tilman B30, 4000 Liège, Belgium; steven.laureys@ulg.ac.be
The results show that patients with devastating motor neurological impairment can have preserved intact cognitive functioning. The locked-in syndrome (LIS; most frequently following a ventro-pontine stroke) is another example of extreme motor impairment. Indeed, patients with LIS are classically tetraplegic and anarthric, but are fully conscious and communicate their thoughts and feelings via eye movements or blinking. Several case reports have shown good cognitive functioning in these patients (for a review see Laureys and colleagues ${ }^{2}$ ). Recently, Schnakers et al have confirmed these results in a cohort of patients with chronic LIS. The neuropsychological battery adapted to eye coded "yes-no" responses detected no deficits in patients with an isolated brainstem lesion, but did show cognitive impairments in patients with additional supratentorial cortical lesions. ${ }^{3}$ In the latter cases, detection of cognitive deficits permitted adaptation of the communication mode in order to fit the preserved cognitive abilities and thus to facilitate communication and to increase the patient's autonomy.

Contrary to LIS caused by an acute brainstem lesion, ALS is a progressive neurodegenerative disease. This implies that, in the later stages, patients with ALS will loose all remaining motor ability to communicate. In such a situation we currently have no way to objectively assess these patients' residual cognitive abilities, or even the presence or absence of consciousness. A solution could be the use of EEG based brain-computer interfaces (also called "thought translation devices"). ${ }^{4}$ Another recently proposed possibility is communication via salivary $\mathrm{pH}$. Indeed, mental imagery of "milk" versus "lemon" is associated with changes in the salivary $\mathrm{pH}$, and the latter can be used as a surrogate "yes-no" response mode. This approach was shown to be successful in a complete locked-in patient with ALS (ie, total immobility including ocular and palpebral movements). ${ }^{5}$

By validating adapted neuropsychological testing and by demonstrating preserved cognition in patients with devastating motor neurological lesions, the study of Lakerveld and colleagues ${ }^{1}$ has important medical-ethical implications. It underscores the right of these patients to autonomy and demonstrates their ability to exercise it, including taking end of life decisions. For patients with no residual motor responses, additional investigations will have to be performed before this ultimate liberty can also be extended to them. This demonstration in ALS of patients' right to autonomy and capability of exercising it will reduce the ethical dilemmas of caregivers but not their responsibilities. Huge challenges remain. Firstly, it will be necessary for caregivers to provide all of the information that is necessary to validly exercise autonomy and then to ask all relevant questions. Moreover, they will have to phrase questions in such terms that they can unambiguously be answered by yes or no. The latter is tantamount to making the complex realm of existence, ideas, feelings and volitions binary. Secondly, many additional conditions need to be fulfilled for autonomy to be full autonomy. Indeed, to quote Harsanyi, "... we have to (...) distinguish between a person's manifest preferences and his true preferences. [...] A person's true preferences are the preferences he would have if he had all the relevant factual information, always reasoned with the greatest possible care, and were in a state of mind most conducive to rational choice". ${ }^{6}$ The beauty of medical and communication-technological progress for patients with severe neurological conditions is that it makes them more and more like the rest of us. As a corollary, we caregivers not only owe them the same respect in terms of their autonomy as all other patients, but we also have to rise to so far seldom attained levels of clarity in matters of life and death.

Funding: Supported by funds from the Belgian "Fonds de la Recherche Scientifique" (FNRS), "Action de Recherche Concertée (ARC) de la Communauté Française", the European Commission and the Mind Science Foundation. Competing interests: None.

J Neurol Neurosurg Psychiatry 2008;79:2. doi:10.1136/jnnp.2007.125294

\section{REFERENCES}

1. Lakerveld J, Kotchoubey B, Kübler A. Cognitive function in patients with late stage amyotrophic lateral sclerosis. J Neurol Neurosurg Psychiatry 2008;79:25-9.

2. Laureys S, Pellas F, Van Eeckhout $P$, et al. The locked-in syndrome: what is it like to be conscious but paralyzed and voiceless? Prog Brain Res 2005;150:495-511.

3. Schnakers C, Majerus S, Goldman S, et al. Cognitive function in the locked in syndrome. J Neurol (in press).

4. Kuebler A, Kotchoubey B. Brain-computer interfaces in the continuum of consciousness. Curr Opin Neurol (in press).

5. Wilhelm B, Jordan M, Birbaumer N. Communication in locked-in syndrome: effects of imagery on salivary pH. Neurology 2006;67:534-5.

6. Harsanyi JC. Morality and the theory of rational behaviour. In: Sen A, Williams B, eds. Utilitarianism and beyond. Cambridge: Cambridge University Press, 1982:55 